\title{
REASONS FOR NOT HAVING AN EARLY ASTEROID MISSION
}

\author{
EDWARD ANDERS \\ University of Chicago
}

Let me first emphasize the area of agreement with Professor Alfvén. I, too, believe that the asteroids are of very great scientific interest; great enough to justify space missions some day. What we differ on is the timing and target selection for these missions (Alfvén and Arrhenius, 1970, and in this volume ${ }^{1}$ ). I look upon space missions as a tremendously expensive way of obtaining scientific data, which should not be attempted until all available alternatives are exhausted. Ground-based research on asteroids and meteorites is nowhere near exhaustion; on the contrary, it is moving at an impressive pace. If we maintain this pace for another decade or two, we will not only have answered most of the questions posed for an early mission, but will be able to come up with a more worthwhile, more informative mission.

Harold Urey once said that meteorites are the only samples of extraterrestrial matter delivered to our doorstep free of charge. Although some people will disagree, I think there is more than a slight chance that most meteorites come from the asteroid belt. It would be tremendously embarrassing to our entire profession if it turned out after a mission to Eros that pieces of Eros (erotic meteorites?) have been reposing in our museums all along. I say "embarrassing" because I think it is well within our powers to trace each group of meteorites to its parent body in the sky. What makes the problem tractable is the small number of objects to be matched up: 6 to 11 meteorite parent bodies and about 7 asteroid families. Each successful match reduces the number of combinations remaining. Let me outline some possible approaches.

\section{ORBITAL CLUES}

Arnold's (1965) Monte Carlo method makes it possible to trace meteorites to their parent bodies, by comparing observed meteorite orbits with computergenerated sets for various possible parent bodies. Wetherill $(1968,1969)$ has made major improvements in the model, and others are undoubtedly feasible. Once a way has been found to treat distant interactions with Jupiter, the model will have reached a degree of realism at which meaningful comparisons with observed meteorite orbits can be made.

${ }^{1}$ See p. 473. 
Observational material is still scarce; the Prairie Network and the Czechoslovak All Sky Camera Network have thus far yielded orbits for only two meteorites. But similar networks are being built in Canada and Germany, and there is hope that progress in this area will quicken. At least several dozen of the older visual orbits appear to be salvageable (Levin and Simonenko, 1969; Millman, 1969) and additional criteria are available for eliminating the remaining 15 to 20 percent doubtful ones in Millman's selection.

It would be premature to claim any identifications on the basis of the present data. But figures 1 to 3 show that different meteorites and asteroids are readily distinguishable from one another on appropriate plots. The Monte

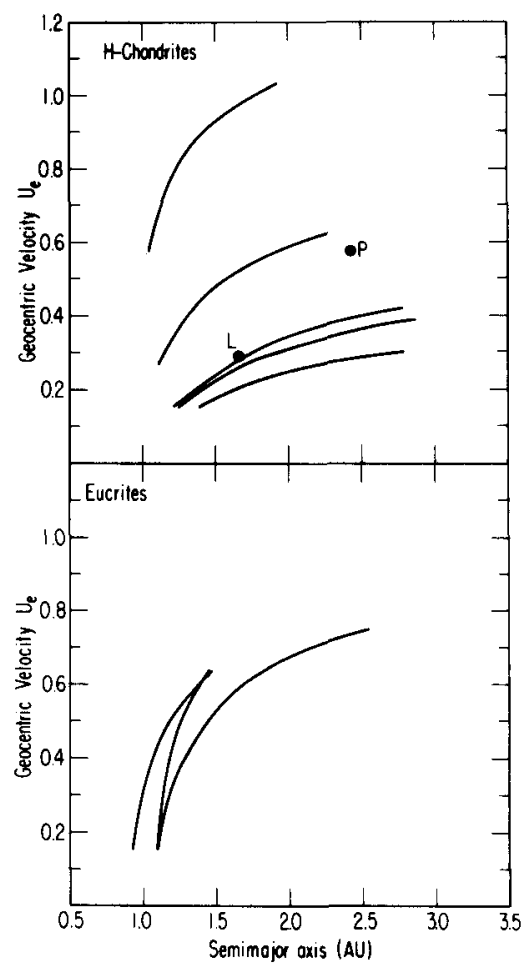

Figure 1.-Observed meteorite orbits. Photographic orbits for Př́bram $P$ and Lost City $L$ are indicated by black circles; visual orbits (Millman, 1969, and personal communication) are indicated by curves representing loci of plausible $U_{e^{-a}}$ combinations. Eucrites appear to have systematically smaller orbits.

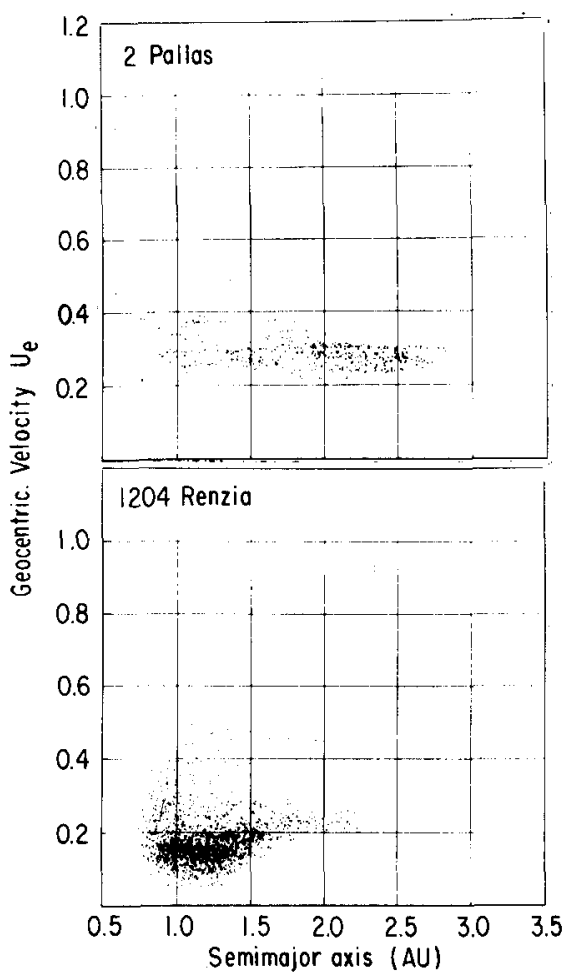

Figure 2.-Monte Carlo orbits of meteorites (Mellick and Anders, unpublished). Both were calculated on the assumption that the inclination drops to a small value whenever the meteorite reaches $a$ near $2.50 \mathrm{AU}(1 / 3$ commensurability with Jupiter), and that the mean life for collisional destruction is $1 \times 10^{7}$ yr. Meteorites from Renzia have systematically smaller $a$ and lower $U_{e}$ than those from Pallas. 


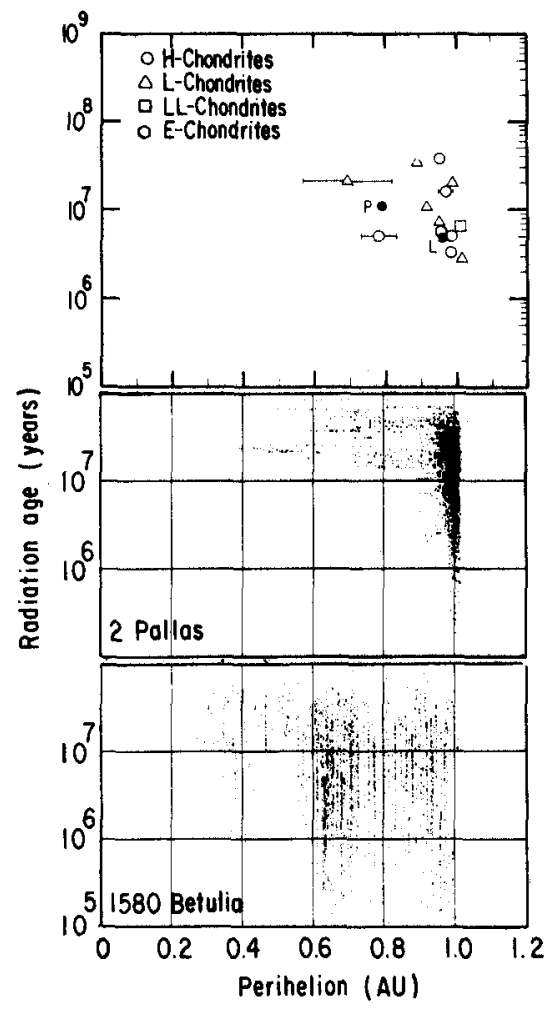

Figure 3.-Radiation age versus perihelion q. Perihelia of chondrites and Pallasderived meteorites tend to be close to $1 \mathrm{AU}$, decreasing slightly with increasing radiation age. This is true of all Mars-crossing asteroid families, as long as older meteorites are eliminated by collisional destruction, with mean life of $10^{7} \mathrm{yr}$. For Betulia and other initially Earth-crossing parent bodies, smaller perihelia appear from the very beginning. Open symbols represent visual orbits; black symbols, photographic orbits of the $\mathrm{H}$-chondrites PYÝbram $P$ and Lost City $L$.

Carlo orbits were calculated on the "optimistic" assumption that geocentric velocity $U$ is occasionally reoriented by Jupiter perturbations when the semimajor axis $a$ reaches a major commensurability (Anders ${ }^{2}$ ). Williams' 3 newly discovered resonances suggest that such an assumption is not grossly unrealistic. As the model is further refined, definite identifications ought to be possible.

Some of the parameters most useful in such comparisons can be obtained from sources other than photographic orbits. Perihelia often can be estimated with surprisingly good accuracy from visual observations. Small perihelia (less than 0.5 to $0.7 \mathrm{AU}$ ) can be inferred from loss of cosmogenic gases. Some meteorite classes (e.g., H-chondrites) show such gas losses more frequently than others; and because they also show a preference for a.m. falls, they probably have orbits with small $a$ and small $q$ (Wänke, 1966).

Geocentric velocities can also be estimated from the ablation loss, which in turn can be determined from measurement of charged particle tracks or cosmogenic radioactivities (Bhandari, 1969).

\footnotetext{
${ }^{2}$ See p. 434

${ }^{3}$ See p. 177.
} 
Finally, some meteorites have radiation ages less than 1 million yr. They are not likely to have experienced any close encounters with Earth prior to impact and thus probably struck Earth from a relatively "unevolved" orbit. Such an orbit should be easy to trace to that of the parent body. If the parent body was Earth-crossing, the two orbits should be identical; if it was Mars-crossing, they should differ by only a single Mars deflection. Sooner or later such a meteorite will be recovered by one of the camera networks. In fact, Lost City, with a radiation age of only 5 million yr, did not intersect Earth's orbit for much of the past 0.5 million yr, according to calculations by Lowrey (1970). Thus it may be suitable for this kind of analysis.

\section{OPTICAL AND CHEMICAL CLUES}

McCord et al. (1970) and Chapman et al. ${ }^{4}$ have shown that reflection spectra of asteroids can provide clues to their composition. Dollfus 5 and Hapke $^{6}$ have used albedo, polarization data, and color indexes (Gehrels, 1970) for this purpose, and have made comparisons with terrestrial, lunar, and meteoritic samples. This is a most promising development. The five known classes of chondrites can be grouped into compositional sequences, on the basis of oxidation state, iron content, $\mathrm{Mg} / \mathrm{Si}$ ratio, etc. Presumably these trends reflect conditions in the solar nebula that varied in some systematic way with distance, time, height above median plane, etc. (Anders, 1971). It will be most interesting to see whether these sequences can also be recognized among the asteroids, and whether they are functions primarily of $a, i$, or radius $R$. The pyroxene band at $0.9 \mu \mathrm{m}$, which is the most distinctive feature in the reflection spectra, will depend both on oxidation state (which determines $\mathrm{Fe}^{2+} / \mathrm{Fe}_{\text {total }}$, and hence the $\mathrm{Fe}^{2+}$ content of the pyroxene) and $\mathrm{Mg} / \mathrm{Si}$ ratio, which determines the pyroxene/olivine ratio.

The degree of fragmentation of an asteroid family (Anders, 1965) can also provide a useful clue. Iron meteorites with a narrow spread of cooling rates apparently come from near the center of their parent body, which must therefore be highly fragmented. Among the chondrites, the proportion of highly recrystallized ones (petrologic type 6) is a clue to degree of fragmentation. For example, only 27 percent of the H-chondrites are type 6, compared to 68 percent of the L-chondrites. Either the L-chondrite parent body was larger and generally hotter, or it was more highly fragmented. (The latter interpretation is more in line with the 520 million yr outgassing event for the L-chondrites; Anders ${ }^{7}$.)

\footnotetext{
${ }^{4}$ See p. 51.

${ }^{5}$ See p. 95 .

${ }^{6}$ See p. 67.

${ }^{7}$ See p. 431.
} 


\section{APPROPRIATE TARGETS FOR AN ASTEROID MISSION}

I have argued in my companion paper ${ }^{8}$ that only high-velocity asteroids, about 10 percent of the total, are potential sources of meteorites. Little or no material reaches us from the remaining 90 percent, including the entire outer half.

If we are successful in matching each meteorite class to its parent body, we will certainly know quite a bit about the high-velocity objects in the inner half of the belt. There will not be much point in sending spacecraft to this relatively well-known part of the asteroid belt.

At this point it is well to consider the nature of the Eros group. There can be no question that Eros and Amor are some of the most accessible asteroids, but they seem to be transient objects. All five have short lifetimes against planetary capture. According to Öpik's (1963) calculations, the lifetimes (in aeons) are as follows:

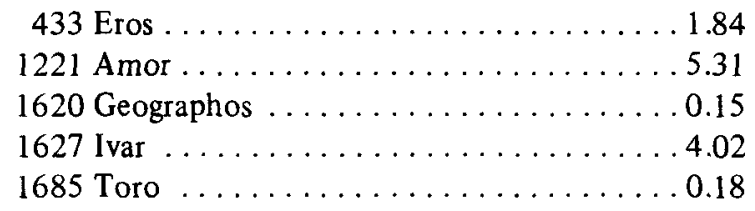

These are probably overestimates, being based on the initial orbit only. Monte Carlo calculations, which consider the change in collision probability after each orbital change in planetary encounters, sometimes give lifetimes up to an order of magnitude shorter for similar objects. In any case, the lifetimes for orbital change are much shorter than those for planetary collision. Thus it is unlikely that these objects formed where they are now found.

We can try to estimate the origin of the three Mars-crossing objects, Making use of the fact that their velocity relative to Mars remains approximately invariant in successive encounters. (The velocity is not strictly invariant because the orbit of Mars has nonzero $e$ and $i$; this causes a slight acceleration of the asteroid at each encounter.) Figure 4 compares $U_{M}$, the velocity relative to a circular orbit at $1.524 \mathrm{AU}$, for the Mars-crossing asteroids.

Eros may have been derived from family 31 after some acceleration by Mars, or from family 5. Amor and Ivar may be members of the Hungaria group at $1.9 \mathrm{AU}$; but this group itself is probably derived from one of the families in the asteroid belt proper: 5,29 , or even 30 . Thus there is not much point in obtaining samples of these stray objects because their original location in the asteroid belt is almost as uncertain as that of meteorites.

If missions with sample return capability are ever sent into the asteroid belt, they should seek to complement the knowledge gained from meteorites. As I

${ }^{8}$ See p. 429 . 


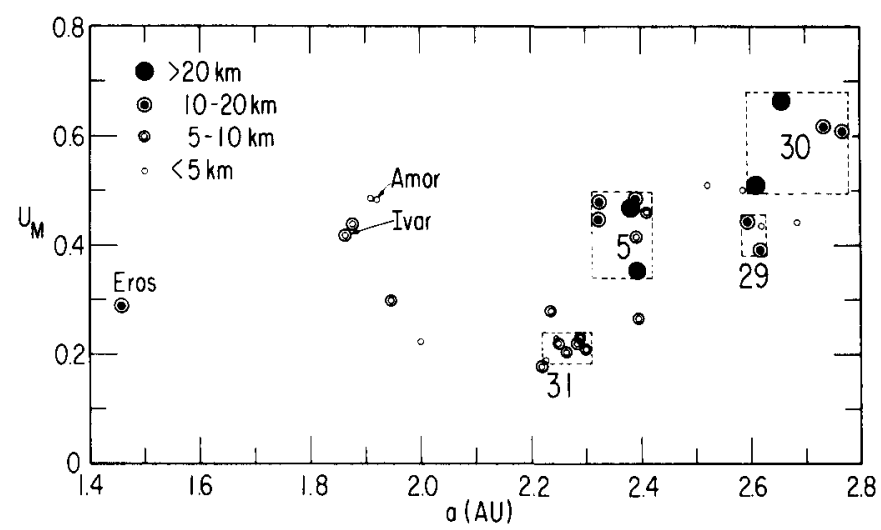

Figure 4.-Mars-crossing asteroids. Eros has nearly the same encounter velocity with Mars $U_{M}$ as families 31 or 5 , and may be a stray member of one of them. Amor and Ivar appear to be related to the Hungaria group at $1.9 \mathrm{AU}$, which in turn may be derived from families 5,29 , or even 30 .

argued in my companion paper, ${ }^{9}$ perhaps as much as 10 percent of the asteroid belt (mainly the high-velocity objects in the inner half) may contribute meteorites to the Earth. The remaining $\geqslant 90$ percent is out of communication with us. If the optimists are right, we may soon gain a good understanding of the "communicating" 10 percent of the belt. Combining this knowledge with telescopic observations, we can then extrapolate at least the gross trends of the "noncommunicating" 90 percent. Some crucial questions will undoubtedly remain when all ground-based studies have been pushed to the limit, and at that stage, perhaps $10 \mathrm{yr}$ from now, further progress will require space missions. We do not know what sort of target will have highest scientific interest at that time: a Trojan, a Hilda group asteroid, a few nearly spherical asteroids (small or large), in the near or far parts of the belt, a few highly irregular objects, a Hirayama family, etc. Any choice we make now is likely to seem trivial or uninformative a decade hence.

If the pessimists are right, even the "communicating" 10 percent of the asteroid belt will be terra incognita to us by the time ground-based work has reached its limits. In that case, a methodical exploration of the asteroid belt may well be justified. Whatever the GNP at that time, it seems certain that the number of missions will be orders of magnitude smaller than the number of asteroids. Targets therefore will have to be selected with very great care. In any event, because proximity is not a critical mission constraint (Bender and Bourke ${ }^{10}$ ), asteroids in the main belt would seem more worthwhile than stray objects such as Eros.

\footnotetext{
${ }^{9}$ See p. 429.

${ }^{10}$ See p. 503.
} 


\section{ACKNOWLEDGMENTS}

The Monte Carlo results in figures 2 and 3 are unpublished data by $P$. J. Mellick. This work was supported in part by AEC Contract AT(11-1)-382 and NASA Grant NGL 14-001-010.

\section{REFERENCES}

Alfvén, H., and Arrhenius, G. 1970, Mission to an Asteroid. Science 167, 139-141.

Anders, E. 1965, Fragmentation History of Asteroids. Icarus 4, 399-408.

Anders, E. 1971, Meteorites and the Early Solar System. Ann. Rev. Astron. Astrophys. 9, $1-34$.

Arnold, J. R. 1965, The Origin of Meteorites as Small Bodies. II. The Model. Astrophys. J. 141, 1536-1547.

Bhandari, N. 1969, On Orbital Velocities of Some Stone Meteorites. Paper presented at the Cosmic Ray Conf. (Delhi).

Gehrels, T. 1970, Photometry of Asteroids. Surfaces and Interiors of Planets and Satellites (ed., A. Dollfus), p. 319. Academic Press, Inc. New York.

Levin, B. Yu., and Simonenko, A. N. 1969, Meteorite Radiants and Orbits. Meteorite Research (ed., P. M. Millman), ch. 46, pp. 552-558. D. Reidel. Dordrecht.

Lowrey, B. E. 1970, Orbital Evolution of Lost City Meteorite. GSFC preprint $\mathrm{X}-644-70-444$.

McCord, T. B., Adams, J. B., and Johnson, T. V. 1970, Asteroid Vesta: Spectral Reflectivity and Compositional Implications. Science 168, 1445-1447.

Millman, P. M. 1969, Astronomical Information on Meteorite Orbits. Meteorite Research (ed., P. M. Millman), ch. 45, pp. 541-551. D. Reidel. Dordrecht.

Öpik, E. J. 1963, Survival of Comet Nuclei and the Asteroids. Advances in Astronomy and Astrophysics 2 (ed., Z. Kopal), pp. 219-262. Academic Press, Inc. New York.

Wänke, H. 1966, Der Mond als Mutterkörper der Bronzit-Chondrite. Z. Naturforsch. A 21 , 93-110.

Wetherill, G. W. 1968, Dynamical Studies of Asteroidal and Cometary Orbits and Their Relation to the Origin of Meteorites. Origin and Distribution of the Elements (ed., L. H. Ahrens), pp. 423-443. Pergamon Press. Oxford.

Wetherill, G. W. 1969, Relationships Between Orbits and Sources of Chondritic Meteorites. Meteorite Research (ed., P. M. Millman), ch. 48, pp. 573-589. D. Reidel. Dordrecht.

\section{DISCUSSION}

KUIPER: As a ground-based observer most of my life, I would naturally sympathize with the point of view of Anders. I think all of us who are working in the laboratory or as astronomers feel that we can do 10 times better or we would not be in the business in the first place. We see many possibilities ahead. I think at the same time it is not right for us to say that the rest must wait until we have made progress. I am all for an accelerated ground-based program for $5 \mathrm{yr}$, but let us not stop the space missions if they can be ready before that.

KIANG: Past attempts at determining the size and shape have had little success, and this is not due to any lack of effort. Here we seem to have an objective that will always evade us however hard we may try from the ground but that can easily be attained by an asteroid mission. Another objective of this nature is the number density of small bodies in the asteroidal belt. At present this is based on brute extrapolation.

DUBIN: I agree with Anders regarding the importance of the ground-based studies of meteorites relative to the understanding of the asteroids, but it appears that the ground-based work cannot resolve the problem in the sense indicated from the Prairie 
Network results shown by McCrosky ${ }^{11}$ earlier. McCrosky showed that most of the bright meteors were of type $\mathrm{A}$ and $\mathrm{C}$ and of low density. The chondrite, Lost City, was one case in a sample of between 100 and 1000 events. In addition, Anders showed that a significant fraction of the mass of the meteoroid, in fact the interesting outer crust, is always ablated during atmospheric entry. I question, accordingly, how these limitations of the sample of meteorites can give a satisfactory model of the early solar nebula and the asteroids.

ALFVÉN: Anders feels that so much information about asteroids could be obtained from the study of meteorites that this, for the time being, ought to substitute for actual exploration in space.

To investigate the evolutionary history of the solar system, it is necessary to pool information from a number of sciences including chemistry, geology, meteoritics, celestial mechanics, and plasma physics. Specialists in any one of these fields may make important contributions but hardly without collaboration with scientists in other fields. This seems not to be generally understood. There have been numerous attempts from astrophysicists to clarify the evolutionary history of the solar system without taking notice of the results of the chemists. Similarly, some competent chemists still tend to draw their conclusions from chemical considerations alone, neglecting the laws of physics. It is obviously unreasonable to write one evolutionary history for physicists and another, completely different, for chemists.

The most important task of the cosmic sciences today is to draw borderlines between speculation and science in its real sense. It is obvious that one of the borders is set by the reach of spacecraft. For example, before the first soft landing on the Moon there were, understandably in view of lacking evidence, many speculations about the chemical composition and physical structure of the lunar surface. We know now that all this had very limited scientific value except as a stimulus toward the actual revealing experiment. For asteroids, a similar situation prevails today, only the uncertainties are wider yet.

During this symposium there has been much discussion about the origin of meteorites. There has been rather extensive support for the view that there is an association between meteorites, comets, and Mars-crossing asteroids, although the arguments for this view are still speculative. Anders, however, believes that the meteorites also come from the main belt asteroids and that certain groups of meteorites should be associated with certain asteroid families. His hypothesis is not demonstrably supported by the laws of celestial mechanics.

UREY: It is my expectation that Eros would have a composition very similar to some meteorite with only minor differences in composition and mineralogy. Also, I suspect that it is a fragment from some violent collision because of its elongated shape. Because of this origin and its very low gravitational field, I believe that it will have no "soil" on its surface and therefore no scooping of soil or drilling of a corc can be made. I strongly suspect that a mission to Eros will be very disappointing to experimenters, the scientific community, NASA, Congress, and the public. I would be delighted to support a mission to Ceres. It may be a fragment of some larger body or it may be a primary object. It may have free rocks and some layer of soil on its surface. Possibly it is a little sister of my lunar-sized bodies or it may be a daughter of them. I would be most interested in seeing a mission to Ceres or the other larger asteroids.

WHIPPLE: I do feel that interest by astronomers in the solar system, particularly in asteroids and smaller bodies, has been negative over the years. Thus I certainly am delighted to see this broad interest of so many coming here to discuss this. It looks to me that it is a healthy attitude toward these problems but I feel this should be supported at a higher level than ever.

However, I have a point about landing on an asteroid. It seems to me that we would like to find a broken-up body so that its surface would give us a historical record,

\footnotetext{
${ }^{11}$ See p. 395.
} 
analogous to geology on the walls of the Grand Canyon. I am sure that the formation processes of asteroids must lead to such historical layers. Perhaps Ceres is not a good choice because its early formations will probably be covered over. On the other hand, I suspect it would be hard to choose an asteroid that would not lead to exciting and unexpected results.

GREENSTADT: Anders' argument against an early asteroid mission and Anders' and Urey's skepticism regarding the value of an Eros mission obtain part of their force from an implicit assumption that such a mission would be created at great expense solely for its own objectives. However, this is not the case. If solar electric and nuclear electric propulsion are to be developed in an orderly way because they are useful for future solat system missions in general, then a proper question is whether Eros, or another Mars-crossing asteroid, would be a suitable target for an early launch with electricpropulsion technology. The choice may be not whether an Eros rendezvous is the best of all asteroid mission objectives, but whether it is best among alternatives for application of early electric-propulsion launches. 